\section{Depression and anxiety as determinants of health-related quality of life in patients with multiple sclerosis - United Arab Emirates}

\author{
Taoufik Alsaadi, ${ }^{1}$ Khadija EI Hammasi, ${ }^{2}$ \\ Tarek M. Shahrour,2 Mustafa Shakra, ${ }^{1}$ \\ Lamya Turkawi, ${ }^{3}$ Wassim Nasreddine, ${ }^{4}$ \\ Seada Kassie, ${ }^{1}$ Mufeed Raoof ${ }^{2}$ \\ 1American Center for Psychiatry and \\ Neurology, Abu Dhabi; 2Department of \\ Psychiatry, Sheikh Khalifa Medical City \\ (SKMC), Abu Dhabi; 3Department of \\ Neurology, Sheikh Khalifa Medical City, \\ Abu Dhabi, UAE; ${ }^{4}$ Department of \\ Neurology, American University of \\ Beirut-Medical Center, Lebanon
}

\begin{abstract}
To study the impact of depression and anxiety on health-related quality of life (HRQOL) in patients with multiple sclerosis (pwMS) in the UAE. All consecutive patients attending the MS clinic over a fourmonth period, October 2014 through February 2015, at Sheikh Khalifa Medical city (SKMC) were asked to complete The World Health Organization quality of life abbreviated scale (WHOQOL-BREF), the Patient Health Questionnaire nine-item (PHQ-9) depression scale, and Generalized Anxiety Disorder seven-item (GAD-7) scale. These last two scales were used to screen for depression and anxiety disorders respectively. The study looked at significant variables associated with HRQOL, using multivariate analysis. Eighty patients were enrolled in the study. Both anxiety and depression had a strong positive correlation with HRQOL in univariate analysis. toms such as fatigue, is found to be the strongest predictor of lower QOL in pwMS; $9,15,16$ although some studies have found anxiety to be a more powerful predictor of QOL. 5 In general, such findings underscore the importance of recognizing comorbid psychiatric disorders in pwMS and call for the need to effectively screen and offer treatment options to these patients.

The epidemiology of psychiatric comorbidity in MS, including its impact on healthrelated quality of life in the UAE remains under-investigated. It can be argued, that in any given neurology practice, the increasing demand to see more patients while spending less time with them could have a causal relationship with comorbid depression and anxiety going undiagnosed/untreated in pwMS. This could have a direct impact on reducing QOL in pwMS. In this study, we sought to determine the primary predictors of HRQOL in pwMS via a detailed analysis encompassing several demographical and disease-related factors.
\end{abstract} However, depression was the strongest predictor of HRQOL in the patients using linear multi-regression analysis. Screening and timely treatment of both anxiety and depression in MS patients should be a recommended policy when managing pwMS to improve their optimal care and ensure better quality of life.

\section{Introduction}

A chronic inflammatory disease of the central nervous system, multiple sclerosis (MS) is shown to have comorbidity with psychiatric illnesses such as depression and anx- iety. ${ }^{1,2}$ Studies have found an obvious interplay between psychiatric comorbidity and reduced health-related quality of life in people with MS.3-5 Additionally, and according to some studies, the prevalence rates of depression and anxiety in MS patients is more than $50 \%$ higher than the general population. 6,7 As a result, MS patients with comorbid depression and anxiety are expected to have reduced health-related quality of life (HRQOL), increased health care utilization, and suicidality. ${ }^{8-13}$ Health-related quality of life is defined as a person's own perception of his or her standard of well-being, both physical and mental, in relation to a chronic illness they suffer from. ${ }^{14}$ Psychiatric comorbidity is shown to have an impact on pwMS and their perceived health-related quality of life. ${ }^{8-10}$ To this effect, recent studies indicated the prevalence and incidence of depression and anxiety in pwMS, calling for improved screening, diagnosis and a more holistic patient care. ${ }^{3-5}$ In most study findings, depression, along with other clinical symp-

\section{Materials and Methods}

A total of 80 consecutive patients attending the MS clinic over a four-month period at Sheikh Khalifa Medical City (SKMC) were asked to enroll in this study. The MS clinic in SKMC is one of the two major tertiary referral clinics in the 2.5 million population of the Emirates of Abu Dhabi, serving close to 400 patients with MS. Only three patients declined to participate. Participating patients were asked to complete the self-administered PHQ-9 and the GAD-7 questionnaires. The English ver-
Correspondence: Taoufik Alsaadi, American Center for Psychiatry and Neurology, Abu Dhabi, United Arab Emirates.

E-mail: t.alsaadi@americancenteruae.com; talsaadi@live.ca

Key words: Multiple sclerosis; Depression; Anxiety; Health-Related Quality of life; UAE.

Conflict of interest: all authors declare no potential conflict of interest.

Ethical approval: The study was granted ethics approval by an authorized Institutional Review Board at the Sheikh Khalifa Medical City, Abu Dhabi, UAE.

Funding: The study did not receive any financial/material support.

Informed consent: Informed consent was obtained from all individual participants included in the study.

Received for publication: 4 August 2017.

Accepted for publication: 11 October 2017.

This work is licensed under a Creative Commons Attribution NonCommercial 4.0 License (CC BY-NC 4.0).

CCopyright T. Alsaadi et al., 2017

Licensee PAGEPress, Italy

Neurology International 2017; 9:7343

doi:10.4081/ni.2017.7343

sions of both questionnaires were used, except for the few Arabic-speaking patients where the Arabic validated versions of both tools were used. 17

The Patient Health Questionnaire nineitem depression scale (PHQ-9), used in this study, is a brief, self-administered tool for the screening and diagnosis of depression. It is based on the nine DSM-IV criteria of depression, as well as being sensitive to change in depression scores over time. A score of $\geq 10$ on the PHQ-9 is indicative of the presence of depression. It can, therefore, be used to monitor response to therapy in this population. The PHQ-9 Depression Scale has been validated in the general population, indicating a prevalence rate of $9.2 \%$ for a current depressive disorder. 18 Undoubtedly, the use of this, and similar other instruments for psychiatric research in MS should be followed by structured psychiatric interviews to confirm clinical diagnoses.

Similarly, to screen for anxiety disorder, the Generalized Anxiety Disorder Scale (GAD-7) was adopted. It consists of a brief, seven-item questionnaire that takes less than three minutes to complete, unlike other screening tools widely used in clinical set- 
tings that are time consuming. A score of $\geq 10$ on the GAD- 7 is indicative of the presence of Generalized Anxiety Disorder. Studies which have used this instrument further support the reliability and internal validity for use in Multiple Sclerosis. ${ }^{19}$

A validated WHOQOL-BREF20 questionnaire was also administered to assess HRQOL. This questionnaire is composed of 26 questions, distributed into four domains. Each domain considers several aspects: A) Physical Domain: pain/discomfort/energy/ fatigue/sleep/rest; B) Psychological Domain: feelings/appearance self-esteem/ memory and concentration; C) Social Relations Domain: social relations/social support/sexual activity; D) Environment Domain: safety/home environment/ finances/leisure/transport/social care.

The patients completed the questionnaires before their assessment in the consultation room. Patients with a confirmed diagnosis of relapsing remitting MS were included, as per the revised MC Donald 2010 criteria, ${ }^{21}$ older than 18 years of age that were either seen for the first time in this clinic, or were seen for a follow up visit. Patients with progressive cognitive deficit that may render them incapable of signing the research consent form were excluded.

Age, sex, number of relapses in the six months prior to the clinic visit, current immunomodulators, EDSS scores, MS duration, PHQ-9, GAD-7 scores, marital status and education level were all recorded. This study was approved by the local institutional review board.

\section{Statistical analysis}

Independent sample t-tests were done to detect significant differences between the categorical variables (Gender, marital status, Educational levels, and relapses within six months) and HRQOL.

Linear regression analysis was carried out to detect association between continuous variables (age, EDSS scores, Duration of MS, PHQ-9, GAD-7) and HRQOL.

Stepwise forward multivariate linear regression analysis was carried out to detect which variables best predict HRQOL. The p-value specified for entry in the multivariate models was 0.05 and the same for removal from the model was 0.1 . The alpha level for all tests of hypotheses of no effect was set to 0.05 .

\section{Results}

A total of 80 patients were seen over the four-month period. The mean age was 35 years (SD 10.60) (Table 1).
Fourteen outof the 80 patients $(17.6 \%)$ reported PHQ-9 scores $\geq 10$ constituting to major depression. Similarly for anxiety scores, 16 patients $(20 \%)$ had scores $\geq 10$ constituting to anxiety disorder. Among all studied variables, the use of antidepressants, PHQ-9, and GAD-7 scores were significantly associated with HRQOL by univariate analysis. The mean HRQOL was 99.5 in those on no antidepressants and 88.2 in those on antidepressants $(\mathrm{P}=0.02)$. Similarly, both PHQ-9 score (R squared $=0.51, \mathrm{P}<0.0001)$ and GAD-7 score ( $\mathrm{R}$ squared $=0.28, \mathrm{P}<0.0001$ ) were significantly linearly correlated with HRQOL in univariate analysis (Figures 1 and 2). However, on multivariate regression analysis, only depression was linearly associated with HRQOL $(\mathrm{P}<0.001)$. When HRQOL was subdivided into the four domains, PHQ-9 score was significantly associated with all these four subdomains. However, the largest linear association occurred between PHQ9 score and Psychological Domain, explaining $43 \%$ of variance in the HRQOL values ( $\mathrm{R}$ squared $=0.43$, $\mathrm{P}<0.0001$ ) (Figure 3).

\section{Discussion}

This study is among the first few in the Arab world that have systematically evaluated the effect of various variables on the overall HRQOL in pwMS. The results are in agreement with previous studies that have identified depression as the main predictor of HRQOL. 9,16,22,23 However, a smaller number of studies have also shown anxiety as another strong predictor for HRQOL. 5,24 Interestingly, the effect of depression and anxiety on physical as well as psychological subdomains were unequal, with depression having the stronger effect on all these subdomains, further suggesting the widespread negative effect of depression, and to a lesser extent anxiety, on multiple domains and accounting for its overall negative effect on the overall HRQOL in pwMS. Similarly, depression, but not anxiety, was the only independent predictor of HRQOL on multi regression analysis. The cause for this discrepancy finding is not clear, and larger, multi-centered studies are needed to confirm this finding.

Surprisingly, and contrary to previous studies, EDSS did not correlate with the overall HRQOL in our patients. This finding may be explained by the low EDSS score of our enrolled patients. The median

Table 1. Sociodemographic information and disease related feature.

\begin{tabular}{|c|c|c|c|}
\hline 6 & $(\mathrm{n}=80), \mathrm{n}(\%)$ & Range, n (\%) & Mean (SD) \\
\hline Age (years) & - & $18-65$ & $35.1(10.5)$ \\
\hline $\begin{array}{l}\text { Sex } \\
\quad \text { Females } \\
\text { Males }\end{array}$ & $\begin{array}{l}52(65) \\
28(35)\end{array}$ & - & $\begin{array}{l}- \\
-\end{array}$ \\
\hline $\begin{array}{l}\text { Marital status } \\
\text { Married } \\
\text { Not married }\end{array}$ & $\begin{array}{l}50(62.5) \\
30(37.5)\end{array}$ & - & - \\
\hline $\begin{array}{l}\text { Education } \\
\text { Lower education } \\
\text { Higher education }\end{array}$ & $\begin{array}{l}33 \text { (41.3) } \\
47(58.7)\end{array}$ & - & - \\
\hline Multiple sclerosis duration since diagnosis (years) & - & $0.1-23$ & $7.8(4.6)$ \\
\hline EDSS score & & $0-6.5$ & $\begin{array}{c}1.7(2.2) \\
\text { Median (0.5) }\end{array}$ \\
\hline $\begin{array}{l}\text { Patients relapsed within } 6 \text { months } \\
\text { No relapse } \\
\text { Relapse }\end{array}$ & $\begin{array}{c}74(92.5) \\
6(7.5)\end{array}$ & - & - \\
\hline $\begin{array}{l}\text { Treatment status } \\
\text { On treatment } \\
\text { Not on treatment }\end{array}$ & $\begin{array}{l}54(67.5) \\
26(32.5)\end{array}$ & - & - \\
\hline $\begin{array}{l}\text { Prescribed antidepressants } \\
\text { Yes } \\
\text { No }\end{array}$ & $\begin{array}{l}5(35.7) \\
9(64.3)\end{array}$ & $\begin{array}{c}58(87.9) \\
8(12.1)\end{array}$ & \\
\hline $\begin{array}{l}\text { Prescribed antianxiety } \\
\text { Yes } \\
\text { No }\end{array}$ & $\begin{array}{c}5(31.3) \\
11(68.7)\end{array}$ & $\begin{array}{c}56(87.5) \\
8(12.5)\end{array}$ & \\
\hline
\end{tabular}

SD, standard deviation; EDSS, Expanded Disability Status Scale. 
EDSS score in our patients was 0.5 , reflecting a mildly disabled population, which could explain this negative finding. Notably, all previous studies reporting positive findings, correlating the strong effect of EDSS to HRQOL, had enrolled patients with higher EDSS scores. For instance, a

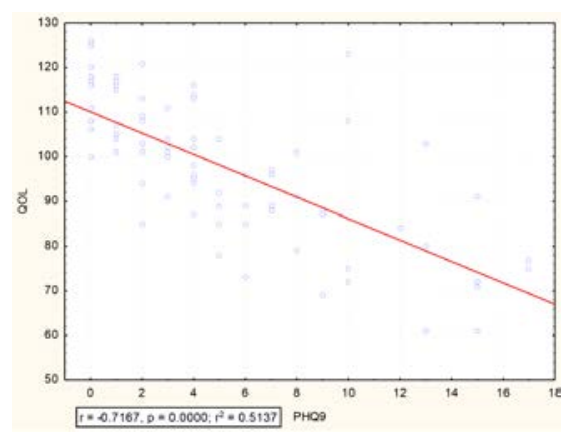

Figure 1. Correlation between Patient Health Questionnaire nine-item and the health-related quality of life rates.

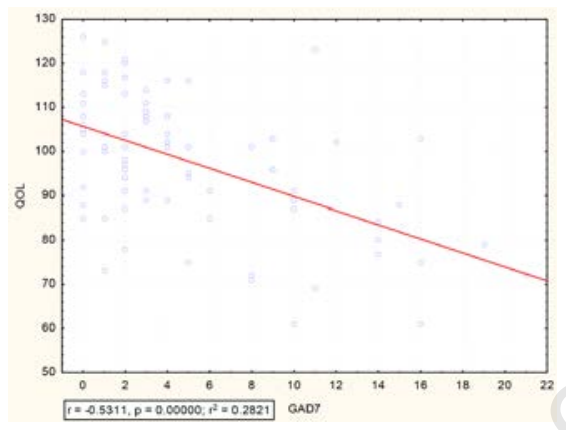

Figure 2. Correlation between Generalized Anxiety Disorder seven-item and the health-related quality of life rates.

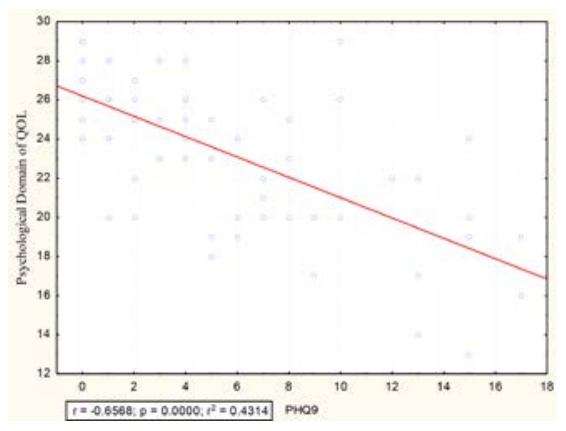

Figure 3. Correlation between psychological subdomain of the health-related quality of life and Patient Health Questionnaire nine-item scores. previous study has found a negative correlation between EDSS and HRQOL, only when the EDSS was larger than a cutoff of 3.25 It is worth pointing out that a previous study found that pwMS are less concerned than their clinicians about physical disability, and that none of the measures of physical disability correlated with the overall HRQOL. ${ }^{26}$ The same conclusion was reached by another study which found that physical disabilities are not predictive of HRQOL in pwMS as compared with age or disease duration. ${ }^{27}$ In addition, a previous study did not find any correlation between the rates of depression and anxiety, which in this study were found to be predictive of the HRQOL, and the EDSS score. ${ }^{28}$ Furthermore, the EDSS, according to the contemporary WHO-ICF model, can only explore the domain of limitations in motor activities. ${ }^{29}$ In this case, scores are assigned based on actual performance during the clinical examination so that EDSS may be less representative and of less significance to the HRQOL.

\section{Limitations}

We realize the limitations of our study. Eighty patients with a considerable degree of demographic heterogeneity took part in the study, and the authors are of the view that a larger, more homogenous sample would have a more statistically significant finding. Such factors may limit generalizability of the results to a wider population. Furthermore, the questionnaire used to measure Health-related Quality of Life, the WHOQOL-Bref, is not typically used in MS HRQOL studies. Nonetheless, other studies that have utilized the same tool have confirmed its validity in assessing several domains in HRQOL. ${ }^{30}$ In general, a larger study from this region is needed to corroborate the findings.

\section{Conclusions}

Future studies should look at using a larger sample size with a more homogeneous group in this region. Replication of the study is warranted using multi-center research including as many MS clinics in the wider MENA region as possible. As supported by this study's findings that corroborate past research findings, it is pertinent to note that clinical attention should be given to the high prevalence of psychiatric comorbidity in MS patients that is shown to have an impact on HRQOL in pwMS. The robust negative impact of depression, and to a lesser degree, anxiety, on the overall quality of life in pwMS should alert neurologists to enhance patient care planning. The utilization of time efficient screening tools should be a standard practice in all MS clinics. Careful screening for these and other related disorders is important to improve the overall quality of life for MS patients.

\section{References}

1. Anne Marie, R. Psychiatric comorbidity in multiple sclerosis: It's not the genes. Mult Scler J 2014;20:1803-5.

2. Haussleiter IS, Brune M, Juckel G. Psychopathology in Multiple Sclerosis: Diagnosis, prevalence, and treatment. Ther Adv Neurol Disord 2009;2:13-29.

3. Anne Marie R, Reingold S, Cohen J, Stuve O, Trojano M, Sorensen PS, Cutter G, Reider N. The incidence and prevalence of psychiatric disorders in multiple sclerosis: A systematic review. Mult Scler J 2015;21:305-17.

4. Huang H, Laursen B, Stenager EN, Stenager E. Psychiatric comorbidity in multiple sclerosis: The risk of depression and anxiety before and after MS diagnosis. Mult Scler J 2016;22:347-53.

5. Salephoor G, Rezaei S, Husseininezhad M. Quality of life in Multiple Sclerosis (MS), and role of fatigue, depression, anxiety, and stress: A bi-center study from North of Iran. Iran J Nurs Midwifery Res 2014;19:593-9.

6. Jones KH, Ford DV, Jones PA, et al. A large-scale study of anxiety and depression in people with multiple sclerosis: A survey via the web portal of the UK MS Register. PLoS One 2012; 7:e41910.

7. McCabe MP, McKern S. Quality of life and multiple sclerosis: Comparison between people with multiple sclerosis and people from the general population. J Clin Psychol Med Settings 2002;9: 287-95.

8. Berrigan LI, Fisk JD, Patten SB, et al. Health-related quality of life in multiple sclerosis: direct and indirect effects of comorbidity. Neurology 2016;86:141724.

9. Garg H, Bush S, Gappmaier E. Associations between MS and disability, functional mobility, depression, and quality of life in people with multiple sclerosis. Int J MS Care 2016;18:71-7.

10. Olascoaga J. Quality of life and multiple sclerosis. Rev Neurol 2010;51:27988.

11. Feinstein A. An examination of suicidal intent in patients with multiple sclerosis. Neurol 2002;59:674-8.

12. Rudick RA, Miller DM. Health-related quality of life in multiple sclerosis: Current evidence, measurement and 
effects of disease severity and treatment. CNS Drugs 2008;22:827-39.

13. Yamout B, Issaa Z, Herlopian A, et al. Predictors of quality of life among multiple sclerosis patents: a comprehensive analysis. Eur J Neurol 2013;20:756-64.

14. Benito-Lion J, Gonzalez JM, RiveraNavarro J, Mitchell AJ. A review of the impact of multiple sclerosis on healthrelated quality of life. Disabil Rehabil 2005; 25: 1291-303.

15. Feinstein A, Magalhaes S, Richard JF, et al. The link between multiple sclerosis and depression. Nat Rev Neurol 2014;10:507-17.

16. Vasconcelos AG, Haase VG, de Paula Lima E, Lana-Peixoto MA. Maintaining quality of life in multiple sclerosis: Fact, fiction, or limited reality? Arq Neurops 2010;5:726-30.

17. Al Sayah F, Ishaque SS, Lau DD, Johnson JA. Health related quality of life measures in Arabic speaking populations: A systematic review on crosscultural adaptation \& measurement properties. Qual Life Res 2013;22:21329.

18. Martin A, Winfried R, Klaiberg A, et al. Validity of the brief Patient Health Questionnaire mood scale (PHQ-9) in the general population. Gen Hosp Psychiatry 2006;28:71-7.

19. Lowe B, Decker O, Muller S, et al. Validation and standardization of the Generalized Anxiety Disorder Screener (GAD-7) in the general population. Med Care 2008;46:266-74.

20. Trompenaars F, Masthoff E, Heck G, et al. Cntent validity, construct validity, and reliability of the WHOQOL-Bref in a population of Dutch adult psychiatric outpatients. Qual Life Res 2005;14:151-60.

21. Polman CH Reingold SC, Banwell B, et al. Diagnostic Criteria for multiple Sclerosis. Ann Neurol 2011;69:292-02.

22. D'Alisa S, Miscio G, Baudo S, et al. Depression is the main determinant of quality of life in multiple sclerosis: a classification-regression (CART) study. Disabil Rehabil 2006;28:307-14.

23. Fruehwald S, Loeffler-Stastka H, Eher $\mathrm{R}$, et al. Depression and quality of life in multiple sclerosis. Acta Neurol Scand 2001;104:257-61.

24. Janssens AC, van Doorn PA, de Boer $\mathrm{JB}$, et al. Anxiety and depression influence the relation between disability status and quality of life in multiple sclerosis. Mult Scler 2003;9:397-403.
25. Kobelt G, Berg J, Lindgren P, et al. Costs and quality of life for patients with multiple sclerosis in Germany. Eur J Health Econ 2006;7:34-44.

26. Rothwell PM, McDowell Z, Wong CK, Dorman PJ. Doctors and patients don't agree: Cross-sectional study of patients' and doctors' perceptions and assessments of disability in multiple sclerosis. Br Med J 1997;314:1580-3.

27. Ford HL, Gerry E, Johnson MH, Tennant A. Health status and quality of life of people with multiple sclerosis. Disabil Rehabil 2001;23:516-21.

28. Alsaadi T, El Hammasi K, Shahrour TM. Prevalence of depression and anxiety among patients with multiple sclerosis attending the MS clinic at Sheikh Khalifa Medical City, UAE: Cross-sectional study. Mult Scler Int 2015;2015: 487159.

29. WHO. International classification of functioning, disability, and health-ICF. Prague Med Rep 2001;109:268-74.

30. Wynia K, Middle B, vanDijk JP, et al. The impact of disabilities on quality of life in people with multiple sclerosis. Mult Scler 2008;14:972-80. 